Short Commentary: Open Access

\title{
Ovarian Cancer Treatment by Suicide Gene Therapy: Beauty of Nanotechnology in Medicine
}

\author{
Abhimanyu Thakur', Mahadi Hasan MA Shahed ${ }^{1 *}$, Aishwarya Roy ${ }^{2}$ and Nabila Sayed ${ }^{3}$ \\ ${ }^{1}$ Department of Biomedical Sciences, City University of Hong Kong, Hong Kong SAR \\ ${ }^{2}$ Department of Pharmaceutical Regulatory Affairs, Parexel Ltd., Karnataka, India \\ ${ }^{3}$ Northern International Medical College and Hospital, Dhaka, Bangladesh
}

*Corresponding author: Dr. Mahadi Hasan MA Shahed, Department of Biomedical Sciences, City University of Hong Kong, 83, Tat Chee Avenue, Hong Kong SAR, E-mail: mhmshahed2@cityu.edu.hk

\begin{abstract}
There has been a global interest worldwide in the recent trend of medical researches, which is basically a merge up between basic biology and engineering tools/technology. Imaging, microfluidics, nanofluidics, surface plasmon resonance (SPR) sensor systems, etc. are the widely used engineering tools in medical researches and has given effective clinical results. The most effective and widely used technology amongst these would be nanotechnology which employs methods like nano drug delivery, nano detection of tumors, assay methods, to detect tumor cells, reach the desired target and kill the tumors. This article lays an insight into the application of nanotechnology to treat ovarian cancer by suicide gene therapy, where in the most promising outcome was delivery of DT-A (Diphtheria toxin suicide protein) encoding DNA with transcriptional regulation targeting tumor cells and gene expression in the ovarian region.
\end{abstract}

\section{Keywords}

Nanotechnology, Biomedical engineering, Ovarian cancer, Suicide gene therapy

Patients with advanced ovarian cancer still live with lack of an effective therapeutic solution; recent researchers have found nanoparticles to serve as a replacement therapy for advanced ovarian cancers. A preclinical test was conducted on mice with ovarian tumour using a nanoparticulate delivery method where polymeric nanoparticle or B-amino ester (a biodegradable cationic molecule) was used to suppress tumor growth by targeting gene expression like MSLN and HE4 to tumor cells [1,2]. However, the conundrum question arises; how effective the therapy is and what underlying mechanism makes it better than other available standard therapeutic paradigm like cisplatin and paclitaxel?

The outcome of the preclinical study suggests that direct administration of DNA encoding DT-A through intraperitoneal (i.p.) method (to target genes highly expressed in ovarian tumor cells like MSLN, HE4) along with transcriptional regulation leads to effective tumor mass reduction along with minimal toxicity to specific tissue and blood while prolonging the life span [3]. The treatment employed since very long for treating cancer of epithelial ovary in its advanced stage is surgical removal of tumour mass then chemotherapy using paclitaxel along with cisplatin or carboplatin which are platinum based therapies. What drawbacks this standard therapeutic regimen has then? [4,5]. Although it showed initial response which was favourable but later on the patients became unresponsive and then re occurrence of the disease is a major issue. So, what could be the futuristic effective solution? The answer is "Gene therapy" which is a promising and effective technique to treat metastatic ovarian cancer as these metastatic tumours are mainly located in peritoneal cavity and hence direct i.p. administration of therapeutic DNA would be an effective treatment. Also the route of delivery would protect the nearby organs from toxic side effects. Another added benefit is once inside the tumour cell its expression can be effectively controlled. The limitation with this concept of treatment is that the above said advantages of DNA therapies still needs to be developed and is the objective of all preclinical and clinical studies [6].

There is a significant question which needs to be answered, what vector should be chosen to deliver the DNA ? Viral vectors were widely in use for treatment in most of the clinical trials for ovarian cancer but the focus has been shifted to use of non-viral vectors. The limitations with the use of viral vectors was the obtained results, there were many reported serious adverse effects. A reported event can be taken as an example, where during a phase I clinical trial, a liposome of cationic nature was used for gene transfer in the treatment of peritoneal tumours which led to a restricted antitumor activity, it was also found that the DNA- vector complex was mainly responsible for all of the reported adverse events at all dose levels. Hence DNA encoding DT-A (diphtheria toxin) and a vector such as h-amino ester, a cationic polymer of biodegradable nature can give the desired results, it has been used also to form a complex and deliver DT-A gene to prostatic cancer cells. The advantage associated with this complex was the potency of the toxin in inhibition of protein synthesis, its capability to reduce tumour mass and finally cell death $[5,6]$. The nanoparticles were formed using DNA and h-amino ester polymer, where only one form of polymer was used, either C32 or C32-117. C32-117 is used as a modified form of $\mathrm{C} 32$ with capping of amine. The advantage of using C32-117 over C-32 is that it has significantly enhanced capability to deliver DNA to the specific ovarian tumour site. The methodology used in this study was development of structural antitumor construct, which is specific to the ovarian region wherein two genes HE4 and MSLN's promoters were employed to regulate and target the expression of DNA in cancerous ovarian cells using in vitro and mouse models. 
The benefit of this technique was evaluated based on the fact that promoter's activity got enhanced significantly in ovarian tumour cells than the other normal cells or tissues. In ovarian cancer cells, Cells devoid of CA125 expression had MSLN and HE4 proteins emerging as the most evident biomarker in them when compared to other proteins. The earlier studies demonstrating the use of DT-A encoded in DNA and its delivery to prostatic tumor cells followed by injection of nanoparticle (using h-aminoester C32) in the intratumoral region led to the evolvement of this strong concept of nanotherapy, as a better and effective technique to deal with progressed stages of ovarian tumour. Problems involving its biodistribution were also solved using i.p. delivery method instead of intravenous (i.v.) and as said earlier most of the metastatic ovarian tumours are located intraperitoneally. The question is can it be practically implemented without any flaws as this needs to have evidence, while treatment involving chemotherapy is an established one? with recent reports of chemotherapy involving i.p. administration proved potential enough as it improved the overall disease free survival rate of the patients having advanced stage ovarian cancer and was recommended as a treatment in January, 2006 to all the physicians by National Cancer Institute (NCI). Three models including xenograft, transgenic and cell implantation were used to evaluate the therapeutic efficacy of nanoparticles. Xenografts are directly injected with nanoparticles while primary ascites obtained from patients was incubated with nanoparticles and it proved the efficiency of nanoparticles in delivering DNA to targeted cancer cells in ovarian region and of human origin [6,7]. Immunosupressed mice were used, where nanoparticles delivered DT-A encoding DNA and due to target of gene expression, suppressed xenografts growth was observed. Apoptosis followed by DT-A expression was observed in MISIIR/ Tag mice where TUNEL assays were done using DT-A nanoparticle injected in tumour cells. It was found that tumour growth was more effectively suppressed in case of MISIIR/Tag mice on treatment with DT-A nanoparticles than on treatment with drugs. While drug treated mice had re-growth of tumour cells, even ID8-Fluc model confirms the effectiveness of DT-A nanotherapy in reduction of tumour load equivalent to combined drug therapy also non-specific toxicity was not observed in DT-A treated mice. ID8-Fluc model which was drug treated showed loss of weight in mice unlike the DT-A treated one. Still this technique could be improvised. How?

By making changes to the nanoparticle formulations for enhancing targeted delivery of DNA to tumour cells also delivery techniques in combined form along with specific promoters for targeting expression which will help in maintenance of healthy tissues. Further limitation to chemotherapeutic treatment is resistance which is again overcome by nanotherapy as DT-A encoding DNA rapidly disrupts protein synthesis and also increases its uptake and expression hence no resistance $[7,8]$.
The future scope for nanoparticles in terms of treatment of ovarian cancer looks promising, this is quite relevant when we evaluate the therapeutic response of DT-A nanoparticles on mouse models (in the absence of non-specific toxicity). The vector C32-117 poly (h-amino ester) is quite effective in DNA delivery to tumor cells followed by i.p administration. The promoter sequences used are also effective in targeting of DNA expression delivered to tumor cells; again resistance is not a limitation with DT-A encoding DNA unlike standard chemotherapeutics. Studies suggest that administration of DT-A nanotherapy is possible and that too for a longer period of time thereby helping in tumor reduction and burden. Opportunities exist in extensive studies which should be aimed on MISIIR/Tag model to evaluate how effective is DT-A nanotherapy as an adjuvant therapy to the above techniques. Also its efficacy rates in treatment of cancer in other parts for example pancreas is to be explored. The existing challenge is that there is no clinical proof or established report employing C32-117 poly (h-aminoester) as a delivery tool. With the recent development in this area of research, an announcement is made by the NCI for sponsoring the preclinical development of this form of therapy at nanotechnology characterisation laboratory which provides further scope for better opportunities.

\section{Conflicts of Interest}

No potential conflicts of interest were disclosed.

\section{References}

1. Hortobagyi GN, Ueno NT, Xia W, Zhang S, Wolf JK, et al. (2001) Cationic liposome-mediated E1A gene transfer to human breast and ovarian cancer cells and its biologic effects: a phase I clinical trial. J Clin Oncol 19: 3422-3433.

2. Peng W, Anderson DG, Bao Y, Padera RF Jr, Langer R, et al. (2007) Nanoparticulate delivery of suicide DNA to murine prostate and prostate tumors. Prostate 67: 855-862.

3. Anderson DG, Peng W, Akinc A, Hossain N, Kohn A, et al. (2004) A polymer library approach to suicide gene therapy for cancer. Proc Natl Acad Sci U S A 101: 16028-16033

4. Sawicki JA, Anderson DG, Langer R (2008) Nanoparticle delivery of suicide DNA for epithelial ovarian cancer therapy. Adv Exp Med Biol 622: 209-219.

5. Zugates GT, Peng W, Zumbuehl A, Jhunjhunwala S, Huang YH, et al. (2007) Rapid optimization of gene delivery by parallel end-modification of poly(betaamino ester)s. Mol Ther 15: 1306-1312.

6. Bingle L, Singleton V, Bingle CD (2002) The putative ovarian tumour marker gene HE4 (WFDC2) is expressed in normal tissues and undergoes complex alternative splicing to yield multiple protein isoforms. Oncogene 21: 2768-2773.

7. Lu H, Li B, Kang Y, Jiang W, Huang Q, et al. (2007) Paclitaxel nanoparticle inhibits growth of ovarian cancer xenografts and enhances lymphatic targeting. Cancer Chemother Pharmacol 59: 175-181.

8. Armstrong DK, Bundy B, Wenzel L, Huang HQ, Baergen R, et al. (2006) Intraperitoneal cisplatin and paclitaxel in ovarian cancer. N Engl J Med 354: 34-43. 